\title{
Secondary Tail Formation and Breakup in Piezoacoustic Inkjet Printing: Femtoliter Droplets Captured in Flight
}

\author{
Arjan Fraters $\odot,{ }^{1}$ Roger Jeurissen, ${ }^{2}$ Marc van den Berg, ${ }^{3}$ Hans Reinten, ${ }^{3}$ Herman Wijshoff, 3,4 \\ Detlef Lohse, ${ }^{1}$ Michel Versluis $\odot,{ }^{1}$ and Tim Segers ${ }^{1, *}$ \\ ${ }^{1}$ Physics of Fluids Group, Max-Planck Center Twente for Complex Fluid Dynamics, Department of Science and \\ Technology, MESA + Institute, and J. M. Burgers Centre for Fluid Dynamics, University of Twente, P.O. Box 217, \\ 7500 AE Enschede, Netherlands \\ ${ }^{2}$ Department of Applied Physics, Eindhoven University of Technology, P.O. Box 513, 5600 MB Eindhoven, \\ Netherlands \\ ${ }^{3}$ Océ Technologies B.V., P.O. Box 101, 5900 MA Venlo, Netherlands \\ ${ }^{4}$ Department of Mechanical Engineering, Eindhoven University of Technology, P.O. Box 513, $5600 \mathrm{MB}$ \\ Eindhoven, Netherlands
}

(Received 4 December 2018; revised manuscript received 27 November 2019; accepted 22 January 2020; published 27 February 2020)

\begin{abstract}
The role of meniscus motion and ink viscosity in the formation of a secondary tail and its breakup are studied experimentally during the picoliter-droplet formation process of a MEMS piezoacoustic inkjet print head using laser-induced 8-ns single-flash stroboscopic imaging with a temporal resolution of $100 \mathrm{~ns}$. It is found that the formation of the secondary tail is driven by meniscus motion and that the secondary tail forms reproducibly between the primary tail and the meniscus in the final microseconds before pinchoff. We demonstrate that the stability of the secondary tail can be controlled through the motion of the meniscus after the primary tail has formed. A 4 times increase in stretching rate results in a 2.2 times increase in the secondary-tail length and a 3 times higher number of femtoliter satellites. Furthermore, as expected for Rayleigh breakup, a $43 \%$ increase in ink viscosity is found to increase the secondary-tail length by $50 \%$. Finally, it is found that, during inkjet printing, the secondary tail cascades into tertiary and quaternary tails. We show that the formation of higher-order tails is irreproducible and therefore driven by noise. The formation of thicker secondary and thinner higher-order tails results in a bimodal satellite size distribution, where the secondary satellites with a volume greater than or equal to $4 \mathrm{fL}$ are located closer to the primarytail droplet, while satellites with a volume less than $4 \mathrm{fL}$ are located closer to the nozzle. The main findings of the present work, that the stability of the secondary tail decreases with a decrease in stretching rate and ink viscosity, can be employed in the inkjet-printing community for waveform design to minimize internal contamination of inkjet printers.
\end{abstract}

DOI: 10.1103/PhysRevApplied.13.024075

\section{INTRODUCTION}

Drop-on-demand (DoD) inkjet printing facilitates highly controlled noncontact deposition of picoliter volumes of material [1-3]. The drop formation can be driven by a piezoelectric actuator, allowing the deposition of fluids and suspensions with a broad range of surface tensions and viscosities [4]. This has enabled inkjet technology to be used not only for classic document printing on paper but also for electronics, e.g., for display fabrication $[5,6]$, for solar-panel printing [7,8], in the life sciences [9-13], and for 3D printing [14]. Extension of the application range demands higher printing precision and higher deposition rates. Thus, the droplet speed, DoD frequency, and nozzle

\footnotetext{
*t.j.segers@utwente.nl
}

integration density must increase, while the droplet size must decrease [15]. To this end, print heads have been developed in silicon, using MEMS technology [16-18].

The geometry of a typical ink channel in a MEMS-based print head developed at Océ Technologies B.V. is shown in Fig. 1(a). The Helmholtz resonance frequency [20] related to the optimum driving waveform of an ink channel, which typically has a value in the range of $100-250 \mathrm{kHz}$, and in this particular study is around $240 \mathrm{kHz}$, can be excited through actuation of a piezoelectric film deposited on a thin flexible membrane. A basic piezo-driving waveform that can be used to produce an ink jet is the monopolar trapezoidal pulse [21] shown in Fig. 1(b). During the rising edge of the pulse (blue lines), the ink is retracted and forms a concave meniscus at the nozzle exit. Moreover, ink is pulled from the ink reservoir during this so-called 
fill-before-fire action, or pull phase [1]. The falling edge of the piezo-driving pulse (red lines) pushes the ink outward in resonance with the channel acoustics, through the nozzle, to form a jet with a head droplet and a long primary tail; see Fig. 1(c) (1-14 $\mu$ s). After pinchoff [22], the primary-tail drop is pulled towards the larger head drop due to surface tension; see Fig. 1(c) $(19-30 \mu \mathrm{s})$. The length of the primary tail increases with an increase in head drop velocity [1]. The long primary tail generated at high droplet velocity may break up due to a Rayleigh-Plateau instability; see Fig. 1(c) (29-30 $\mu$ s). In the ideal case, the primary tail and its primary satellites merge fully with the head droplet before the substrate is reached. However, at high jet velocities the velocity of the primary satellites is substantially lower than that of the head droplet, leading to differences in the arrival times of the droplets at the substrate. These differences, in combination with movement of the substrate with respect to the print head, result in misaligned deposition, leading to decreased print quality $[1,23]$.

A known solution to this problem is the use of a multicomponent piezo-driving waveform of the type described in Refs. [23-25]. Figure 1(b) (lower part) presents the multicomponent pulse that is used in this study, with the delay time $\Delta t$ of the pulse's second actuation as a control parameter. This type of waveform decreases the length of the primary tail at pinchoff and at the same time increases the velocity of the tail droplet; see Fig. 1(d). The additional component of the multicomponent pulse shown in Fig. 1(b) first strongly retracts the meniscus to thin the primary tail [see the difference between Figs. 1(c) and 1(d)] at $5 \mu \mathrm{s}$, and thereby speeds up the contraction of the primary tail after pinchoff. Subsequently, it pushes the meniscus far out of the nozzle, out of resonance with the channel acoustics and in two steps, to prevent the ejection of a droplet; see Fig. 1(d) from 8 to $12 \mu \mathrm{s}$. As a result, the tail droplet is formed at a larger distance from the nozzle, thereby shortening the primary-tail length. Moreover, the head droplet is slowed down more at the beginning of the drop-formation process and less towards pinchoff, which causes the distance from the head droplet to the nozzle exit at pinchoff to be smaller. This difference in head-droplet position, which is visible in Figs. 1(c) and 1(d) at $16 \mu \mathrm{s}$, again leads to a shorter primary-tail length at pinchoff, while the head-drop velocity at a later instance in time (26-29 $\mu \mathrm{s})$ remains the same; see the parallel green lines. Apart from the advantage of a shorter primary tail that contracts faster, the use of this multicomponent pulse has the disadvantage of generating substantially larger amounts of micrometer-sized satellite droplets, called secondary (a)

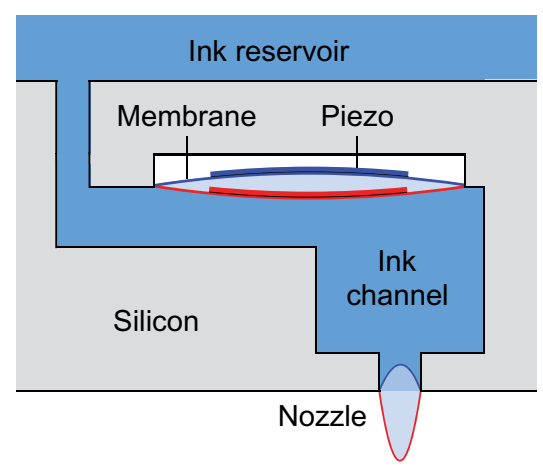

(b)

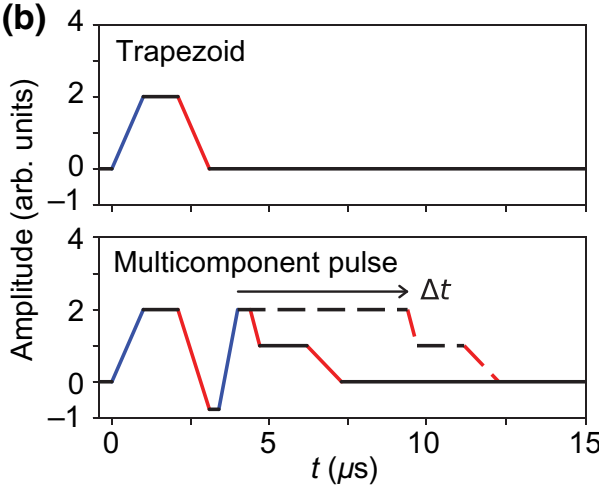

(c)

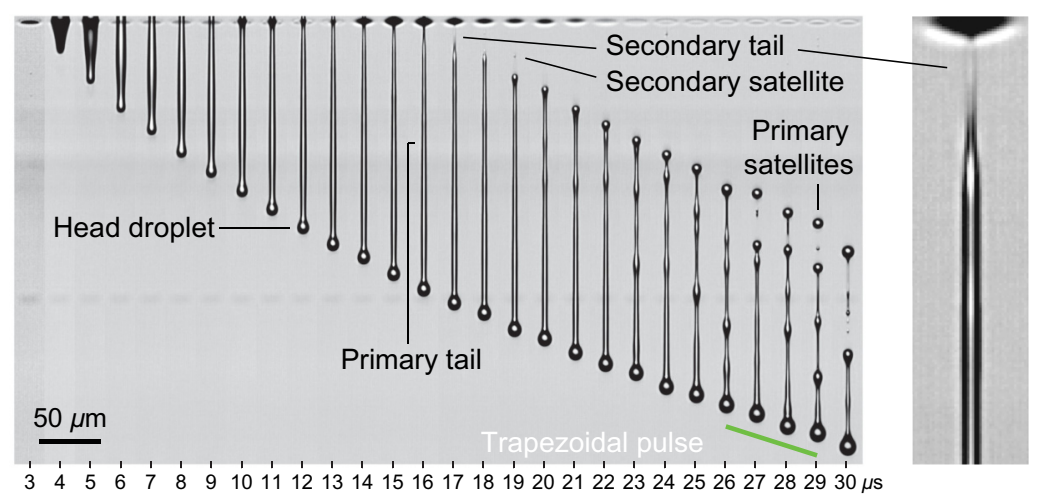

(d)

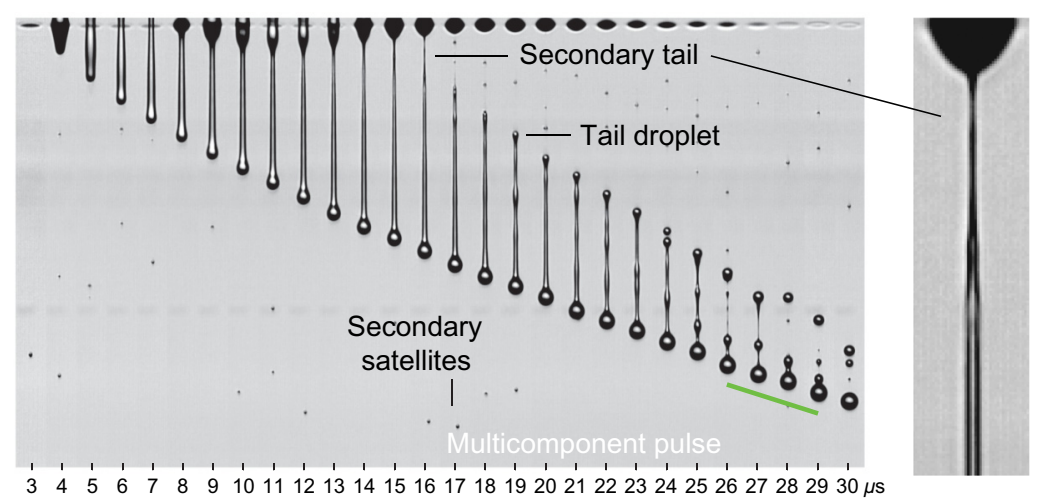

FIG. 1. (a) Schematic drawing of an ink channel in a MEMS-based piezoacoustic print head. (b) Trapezoidal and multicomponent piezo-driving pulses. (c) Droplet formation driven by a trapezoidal pulse. (d) The length of the primary tail can be decreased through the use of a multicomponent pulse, while the droplet velocity remains the same (parallel green lines). However, the amount of secondary satellites is increased dramatically. The imaging technique employed, iLIF [19], is described in Sec. II B. 
satellites; compare Figs. 1(c) and 1(d). The secondary satellites have little inertia and are therefore easily dragged along by the local airflows induced by the fast-moving components of the printer machine. The resulting mist of secondary satellite droplets settles throughout the machine, thereby contaminating the inkjet printer.

Secondary satellites result from the breakup of a secondary tail that forms microseconds before pinchoff between the meniscus and the primary tail $[1,26]$; see Figs. 1(c) and 1(d). The secondary-tail formation starts with the appearance of a neck between the meniscus and the primary tail due to flow reversal in the nozzle [27]. The radius of curvature of the neck is small compared with that of the meniscus, which results in a large pressure gradient that drives a flow of ink from the neck towards the meniscus [1]. As a result, the neck thins and a secondary tail forms $[19,26,28]$ that is stretched, and thus stabilized $[29,30]$, by the opposing movement of the meniscus and the primary-tail drop, to a final radius on the order of $1 \mu \mathrm{m}$; see Figs. 1(c) and 1(d) (15-18 $\mu$ s).

To date, little is known about the influence of the ink properties and the meniscus motion on secondary-tail formation and breakup, and about how these can be optimized to minimize the number of secondary satellites in dropon-demand inkjet printing. Pinchoff studies in continuousink-jet and dripping-drop experiments have revealed fluid structures named "microthreads" and "secondary necks" that appear to be similar to the secondary tails observed in drop-on-demand inkjet printing [31-33]. A pinchoff will eventually evolve according to the Eggers similarity solution [34], but this solution is unstable to a finitesize perturbation [33,35]. For pure fluids [36,37], a large enough perturbation disturbs the pinchoff, which results in a cascade, i.e., in a much thinner thread that converges to the similarity solution again [38]. The perturbation is usually the result of noise: disturbances beyond the control of the experimenter, such as thermal noise, resulting in a stochastic cascade of ever thinner tails. The stochastic nature can be observed from the random locations of the micrometer-sized satellites in the stroboscopically imaged drop-formation processes shown in Figs. 1(c) and 1(d). However, note that the location of the tail droplet is highly reproducible over time, from which it can be concluded that secondary-tail formation in inkjet printing is triggered by the reproducible meniscus motion, and not by noise. Therefore, secondary-tail formation can be studied using stroboscopic imaging techniques.

It has been shown that the final lengths of microthreads and secondary necks increase with an increase in the liquid viscosity $\eta$. The increase in thread length relates to an increase in the viscous length scale $L_{\eta}=\eta^{2} / \rho \gamma$ below which viscous damping plays a dominant role, as compared with surface-tension-driven inertia in pinchoff and Rayleigh-Plateau breakup [32,39]. Therefore we expect that the secondary-tail length in piezo-driven drop-on-demand inkjet printing will also increase with an increase in ink viscosity and that it will decrease with increasing noise amplitude. Tertiary and higher-order necks in the final stage of the breakup of an ink jet have never been observed. This may be partly due to the challenges involved in the visualization of submicrometer threads that are stretched at rates of the order of $10 \mathrm{~m} / \mathrm{s}$. Moreover, the inkjet nozzle diameter is typically one order of magnitude smaller than that of the nozzles employed in the present studies in the literature on microthread and neck formation.

In the present work, the details of secondary-tail formation and breakup are visualized with nanosecond and micrometer precision. The aim is to determine how two control parameters in inkjet printing, i.e., meniscus motion and ink viscosity, affect the stability of the secondary tail. Control over the stability of the secondary tail is pursued in order to minimize the number and volume of the secondary satellites that form after secondary-tail breakup, in order to limit internal contamination of inkjet printers. The imaging challenge is solved by employing single-flash stroboscopic laser-induced fluorescence imaging [19] (iLIF). iLIF was introduced to allow exposure times below $10 \mathrm{~ns}$ with high-intensity incoherent ultrashort light flashes. First, secondary-tail formation is studied for a range of meniscus motions by using a trapezoidal pulse and a multicomponent pulse. Second, secondary-tail formation is studied for two different ink viscosities to verify the hypothesized decrease in secondary-tail length with decrease in ink viscosity. Finally, high-speed imaging is used to reveal, in the context of picoliter inkjet printing, the stochastic cascade of the secondary tail into higher-order tails.

\section{EXPERIMENTAL METHODS}

\section{A. Print head and ink}

The print head used in this study has cylindrical nozzles with a diameter of $14 \mu \mathrm{m}$ and a length of $10 \mu \mathrm{m}$; see Fig. 1(a). Droplets with an approximate volume of $1 \mathrm{pL}$ $(6.2 \mu \mathrm{m}$ radius) are produced with a velocity of $6.8 \mathrm{~m} / \mathrm{s}$, measured at an axial distance of $500 \mu \mathrm{m}$ from the nozzle. Isobornyl acrylate (IBOA, Sigma Aldrich) is used as a model ink, as it is a single-component liquid with a viscosity and surface tension similar to those of the more complex inks that are used in commercial inkjet printing, i.e., $7 \mathrm{mPa}$ and $35 \mathrm{mN} / \mathrm{m}$ at $25^{\circ} \mathrm{C}$. The viscosity and surface tension of the IBOA are measured using a parallelplate rheometer (Anton Paar, MCR 302) and a bubblepressure tensiometer (Sita T60), respectively. Resistive heating elements and temperature sensors are incorporated throughout the print head to accurately control and monitor the ink temperature. The default operating temperature and corresponding ink viscosity and surface tension are $20.9 \pm 0.2^{\circ} \mathrm{C}, 8.02 \pm 0.06 \mathrm{mPa} \mathrm{s}$, and $35.7 \pm 0.1 \mathrm{mN} / \mathrm{m}$, respectively. 


\section{B. Imaging setup}

The single-flash stroboscopic imaging setup, described in detail by van der Bos et al. [28], consists of a microscope connected to a $20 \times$ magnifying objective (Edmund Optics, 59878, M-Plan-APO, NA 0.42) and to a $1 \times-$ magnification tube lens (InfiniTube ${ }^{\mathrm{TM}}$ ). A particle image velocimetry camera (PCO Sensicam QE) is used to capture images and is controlled from a personal computer. The resulting optical resolution is $362 \mathrm{~nm} /$ pixel. To prevent motion blur, illumination is provided by a laser-inducedfluorescence system [19] consisting of a 7-ns pulsed laser (Litron Nano-S, dual-cavity Nd: YAG, $\lambda=532 \mathrm{~nm}, 65$ $\mathrm{mJ}$ ) that illuminates a fluorescent plate in a highly efficient diffuser (Lavision, Parts No. 1108417 and 1003144), resulting in an incoherent 8-ns illumination pulse with a mean wavelength of $577 \mathrm{~nm}$. A lens with a numerical aperture equal to that of the microscope objective condenses the light pulse onto the imaging plane of the microscope. The timing of the illumination flash with respect to the start of the piezo-driving waveform is varied with nanosecond precision through the use of a programmable pulse-delay generator (Berkeley Nucleonics Corp., BNC 575).

\section{Experimental procedure}

For each experimental setting, two image sequences are captured. First, the formation of the secondary tail and the corresponding meniscus motion are stroboscopically imaged at a temporal resolution of $100 \mathrm{~ns}$ over a duration of $30 \mu \mathrm{s}$, starting from the beginning of the piezo-actuation pulse. Secondly, the size distribution of the secondary satellites is imaged by capturing images at a constant delay of $23 \mu$ s with respect to the start of the actuation pulse. In all experiments, the secondary tail breaks up within this $23 \mu \mathrm{s}$, and in almost every image the primary tail is still intact. Since the breakup of the secondary tail is random and highly sensitive to noise, 1000 images of 1000 different drop formations are captured for each experimental setting. The print head is operated at a continuous jetting frequency of $10 \mathrm{kHz}$, and every 6000th droplet formation is imaged to allow sufficient data-transfer time to the personal computer.

In the first experiment, the secondary-tail formation and secondary-satellite size distribution for a trapezoidal pulse and a multicomponent pulse are compared, primarily to study the effect of the amplitude of the meniscus motion on secondary-tail formation. Both of the piezo-driving waveforms are plotted in Fig. 1(b). In this experiment, a multicomponent pulse with $\Delta t=0.4 \mu \mathrm{s}$ is used.

In the second experiment, the $\Delta t$ of the multicomponent pulse is varied over a range from 0.4 to $9.4 \mu \mathrm{s}$ in steps of $1 \mu \mathrm{s}$, primarily to determine the influence of the timing of the meniscus retraction on secondary-tail formation. To extend the $\Delta t$ range, a second measurement series is performed for a $\Delta t$ range from 0.4 to $14.4 \mu \mathrm{s}$ in steps of
$1 \mu \mathrm{s}$. However, only 100 images of the secondary satellite droplets are captured for each $\Delta t$ setting, instead of 1000 in the first case.

In the third experiment, the role of ink viscosity in secondary-tail formation and secondary-satellite size is investigated. To this end, the ink temperature is increased from $20.9 \pm 0.2^{\circ} \mathrm{C}$ to $32.0 \pm 0.4{ }^{\circ} \mathrm{C}$. The ink viscosity thereby decreases from $8.02 \pm 0.06 \mathrm{mPa} \mathrm{s}$ to $5.58 \pm$ $0.07 \mathrm{mPa}$ s. For both viscosities, droplets are generated using a multicomponent pulse with $\Delta t=0.4 \mu \mathrm{s}$. The ink temperature has significantly less influence on the surface tension; it is measured to be $35.7 \pm 0.1 \mathrm{mN} / \mathrm{m}$ and $32.8 \pm 0.1 \mathrm{mN} / \mathrm{m}$ at $20.9^{\circ} \mathrm{C}$ and $32.0^{\circ} \mathrm{C}$, respectively.

\section{Image analysis}

The length $L_{2 t}$ of the secondary tail is measured as a function of time from the image sequences captured of the full drop-formation processes with the different driving waveforms, i.e., a trapezoidal pulse and multicomponent pulses with different delay times $\Delta t$, and for different viscosities. The breakup time is measured visually from the image sequences as the start of the time interval over which the state of the secondary tail is either broken up or undefined, where "undefined" means that it cannot be visually determined whether or not the secondary tail has broken up. The uncertainty in the breakup time is set to the length of this time interval. The maximum outward meniscus position $y_{m}$ and the location of the end of the tail drop $y_{\mathrm{TD}}$ are measured from the images; see Fig. 2(a). $y_{\mathrm{TD}}$ is defined as the inflection point in the axial direction at the transition from the tail drop to the secondary tail. The secondary-tail diameter is on the order of the diffraction limit of the imaging system and can therefore not be quantitatively measured.

The secondary-satellite size distribution is detected from the image sequences captured at a constant delay of $23 \mu \mathrm{s}$ with respect to the start of the actuation pulse using an image-processing algorithm programmed in the Python programming language [40]. The main steps of the imageprocessing algorithm are summarized in Figs. 2(b)-2(e). First, in each raw image [Fig. 2(b)], the background intensity is equalized in the axial direction. To this end, for each pixel row, the median pixel intensity of the background is calculated, and for each image the maximum of these median values is determined. Then, the intensity of each pixel is multiplied by the image's maximum rowmedian background intensity divided by the local rowmedian background intensity. Subsequently, the images in each sequence are cropped according to a region of interest (ROI) that is chosen such that in each image the secondary satellites are located within the ROI. This is illustrated in Fig. 2(c). Then, for each pixel row within a cropped image, the minimum intensity value is found to determine the minimum-intensity profile; see the blue 
(a)

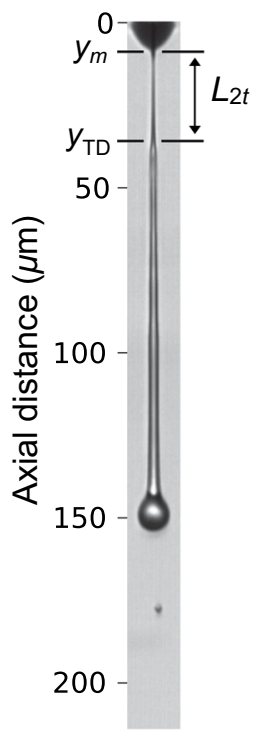

(b)

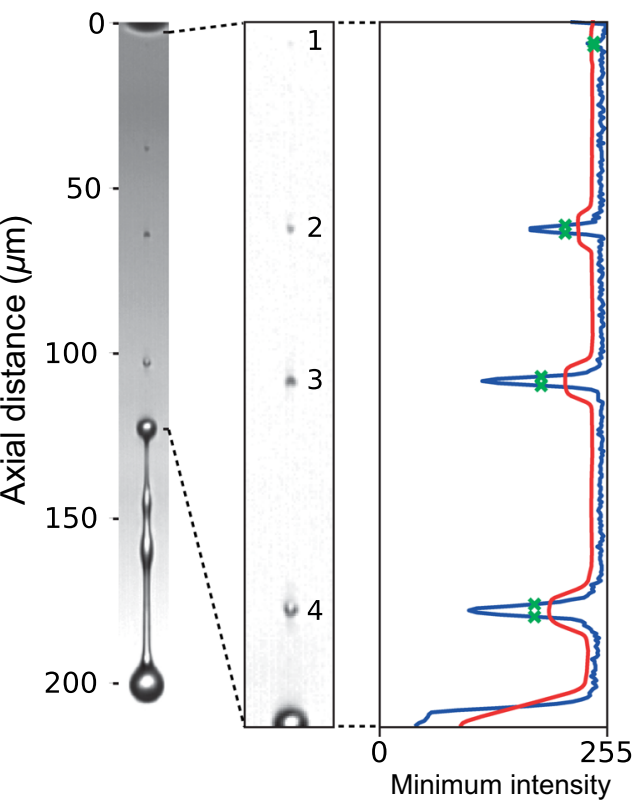

(e)

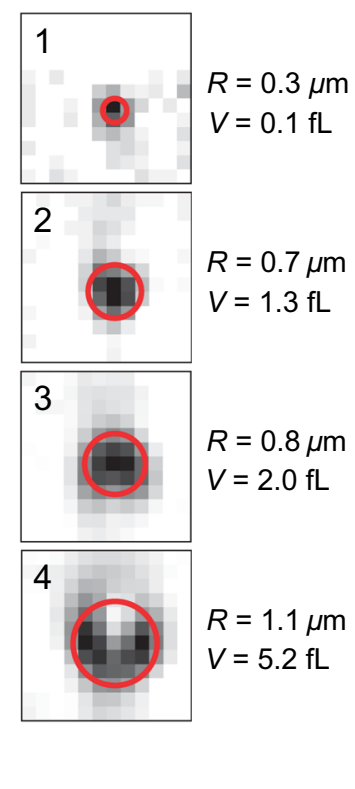

FIG. 2. (a) The secondary-tail length $L_{2 t}$ is measured from the inflection point in the axial direction at the transition from the tail drop to the secondary tail $y_{\mathrm{TD}}$ minus the meniscus position $y_{m}$. (b) From the droplet-formation images, the secondary-satellite position and size distribution are obtained. (c) First, the background intensity is equalized in the axial direction, and the images are cropped. (d) Second, the minimum intensity profile is obtained and used to measure the secondary-satellite sizes. (e) Examples of droplet sizes obtained (red circles). solid line in Fig. 2(d). The satellites are detected in this minimum-intensity profile using a threshold based on the minimum-intensity profile. The minimum-intensity profile is smoothed using the SCIPY SAVGOL filter of first order with a window length of 0.05 times the total profile length. The satellite detection threshold is set as the difference of the smoothed profile intensity values and the median of the profile intensity values, multiplied by 0.8 to prevent false negatives, and lowered by 10 on a scale of $0-255$ to prevent false positives due to background noise; see the solid red line in Fig. 2(d). The size of the detected satellites is measured from the width of the minimum-intensity profile at half the distance between the minimum peak intensity and the median of the minimum-intensity profile; see the green crosses in Fig. 2(d). Around the center location of the detected satellite droplets, this edge detection method is applied once more in the lateral direction. The satellitedroplet size is then set to the minimum obtained in both directions; see the red circles in Fig. 2(e). In the few images in which the rear end of the primary tail is already broken up and a primary satellite appears within the ROI, the primary satellite is removed from the results based on its volume. For the print head employed, there is a clear division between primary and secondary satellites at a volume of $45 \mathrm{fL}$ in the satellite size distribution, i.e., primary satellites are filtered out by excluding satellites with a volume greater than or equal to $45 \mathrm{fL}$.

Figure 2(e) demonstrates the imaging challenge involved in the Mie-scattering-dominated [41] imaging regime of micrometer-sized femtoliter droplets. The scattered light intensity is highly dependent on both the size of the satellite droplets and their position within the depth of field. Moreover, the minimum-intensity profile depends on the background illumination intensity, and so does the detected droplet radius. Satellite droplet sizes measured using the minimum-intensity-profile method can therefore only be used to compare relative numbers. On top of that, all experiments in the same parameter study need to be performed in a single measurement series, using the exact same focusing and illumination conditions.

A second sizing method that allows backgroundintensity-independent sizing of the droplets, based on the inflection point of the angle-averaged intensity profile of a detected satellite droplet [28], is also employed to analyze the captured images. However, its sizing results are found to be inferior to the minimum-intensity-profile method described above due to the nonaxisymmetric pixel intensity of some of the detected droplets [Fig. 2(e-4)]. Therefore, only the results obtained using the minimumintensity-profile method are presented here.

\section{RESULTS}

\section{A. Trapezoidal pulse versus multicomponent pulse}

Figure 3(a) shows an image sequence for secondarytail formation and breakup with a trapezoidal pulse with an interframe time of $1 \mu \mathrm{s}$. Figure 3(b) presents a similar image sequence for a multicomponent pulse with $\Delta t=0.4 \mu \mathrm{s}$.

Figure 3(c) displays the maximum outward meniscus position $y_{m}$ (square symbols) and the tail-drop position $y_{\mathrm{TD}}$ (triangular symbols) as a function of time. The dashed vertical lines mark the appearance of the secondary tail, i.e., the moment at which the neck has thinned down sufficiently for its length to become measurable, while the solid vertical lines indicate the occurrence of secondary-tail 
(a) Trapezoidal pulse

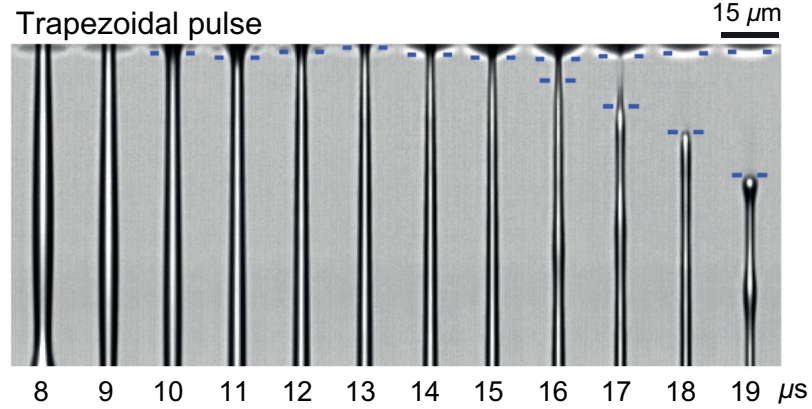

(b) Multicomponent pulse

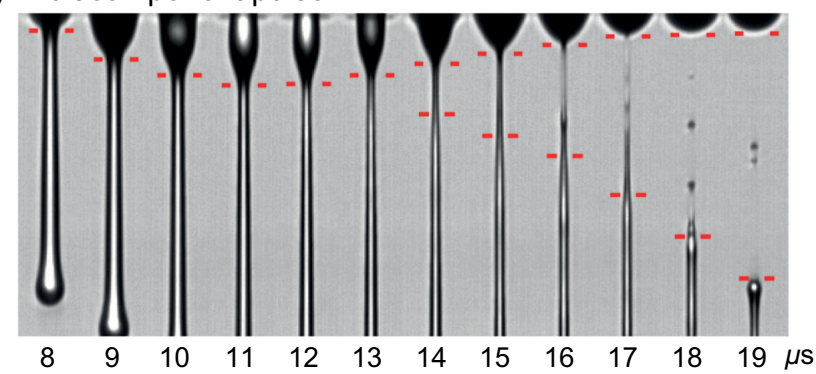

(i) Trapezoidal pulse

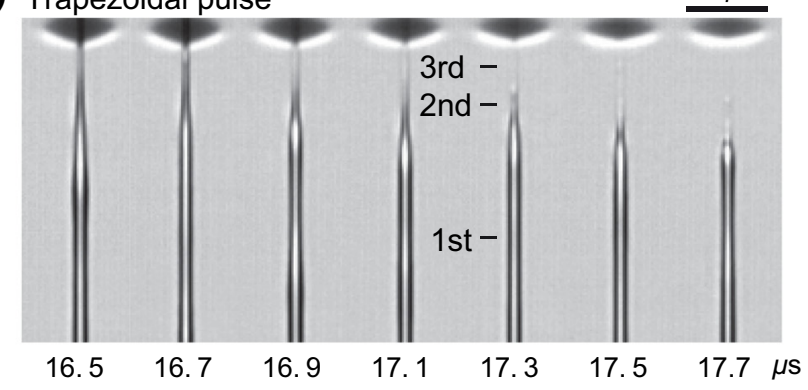

(j) Multicomponent pulse

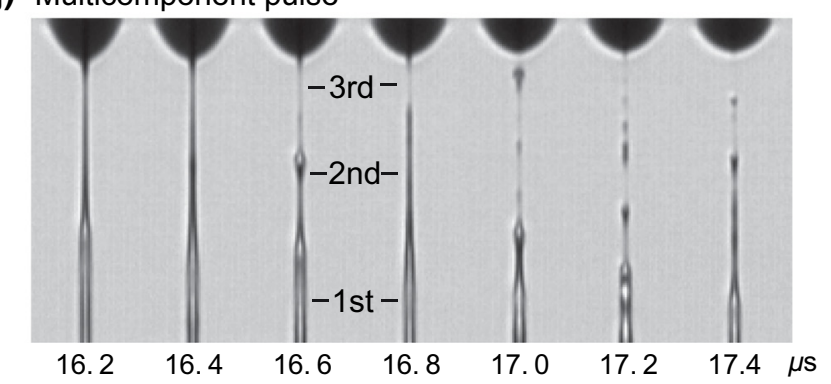

(c)
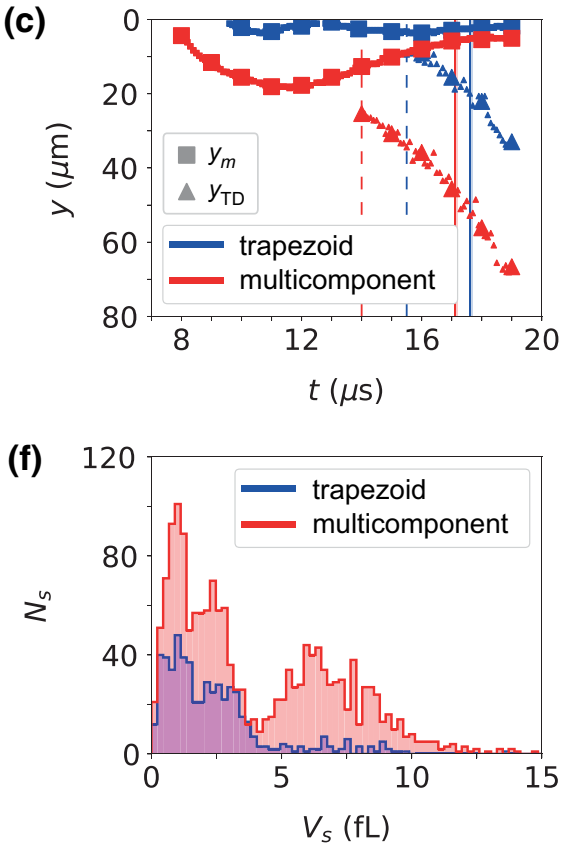

(d)

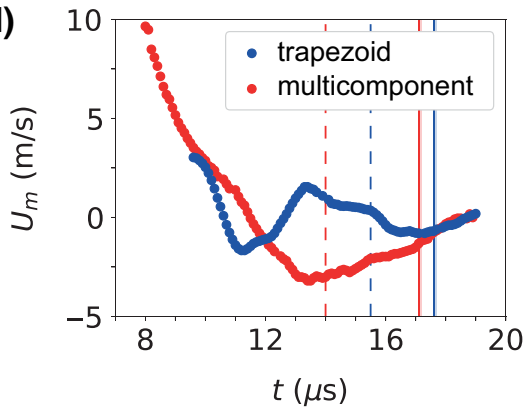

(g)

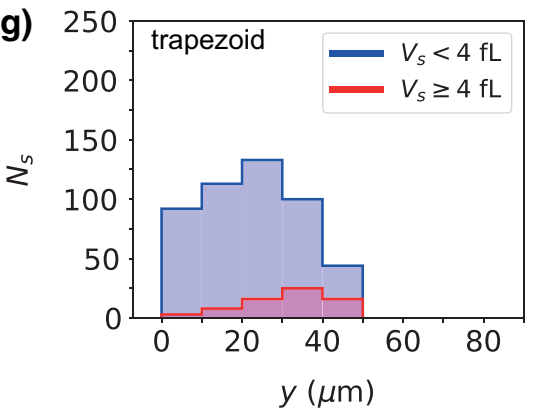

(e)

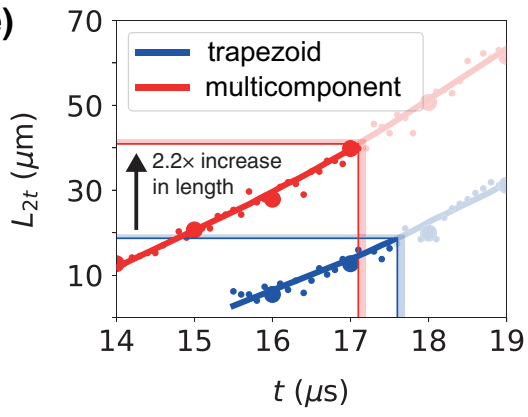

(h)

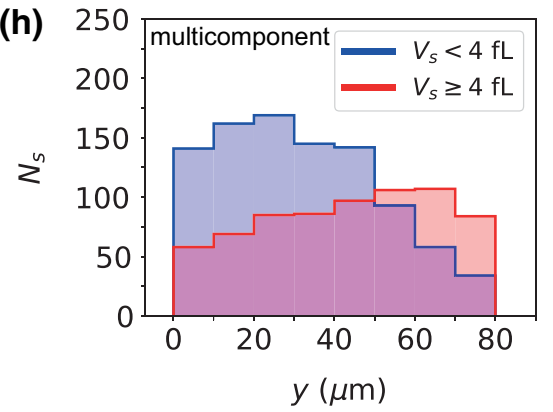

FIG. 3. Secondary-tail formation and breakup for (a) a trapezoidal pulse and (b) a multicomponent pulse with $\Delta t=0.4 \mu$ s. The temporal resolution is $1 \mu \mathrm{s}$. (c) Meniscus position $y_{m}$ and tail-drop position $y_{\mathrm{TD}}$ as a function of time. The large symbols in (c),(e) correspond to the images in (a),(b). The dashed and solid vertical lines in (c), (d), and (e) indicate the appearance and breakup, respectively, of the secondary tail. The translucent bars represent the uncertainty in the breakup time. (d) Meniscus velocity as a function of time. (e) Secondary-tail length as a function of time. After breakup, indicated by the horizontal and vertical solid lines, the evolution of the position of the end of the primary tail is indicated by the translucent data points. (f) Secondary-satellite volume distributions. Position distribution of secondary satellites with a volume less than $4 \mathrm{fL}$, and that of secondary satellites with a volume greater than or equal to $4 \mathrm{fL}$, both generated using $(\mathrm{g})$ the trapezoidal pulse and $(\mathrm{h})$ the multicomponent pulse. Image sequence for the secondary tail with a temporal resolution of $0.2 \mu \mathrm{s}$ for (i) the trapezoidal pulse and (j) the multicomponent pulse with $\Delta t=0.4 \mu \mathrm{s}$ of Sec. III B; see also Figs. 5 and 7 for more details.

breakup. The meniscus-position plot for the multicomponent pulse has a maximum value 5.1 times larger than that of the trapezoidal pulse, resulting in a meniscus velocity approximately 3 times higher; see Fig. 3(d). Moreover, for the multicomponent pulse, the meniscus velocity during the lifetime of the secondary tail is approximately 4 times 
as large when it is negative, i.e., retracting. For the trapezoidal pulse, the tail-drop formation starts at a later time, and closer to the nozzle than that for the multicomponent pulse; see Fig. 3(c). This can also be observed in Fig. 3(e), where the secondary-tail length $L_{2 t}$ is plotted as a function of time. At the moment of secondary-tail breakup (solid vertical lines), $L_{2 t}$ is 2.2 times longer for the multicomponent pulse than for the trapezoidal pulse. Furthermore, note that the lifetime of the secondary tail formed using the multicomponent pulse is approximately $40 \%$ longer than that for the trapezoidal pulse.

For both driving pulses, the size distribution of the secondary satellites is shown in Fig. 3(f). Droplet formation driven by the multicomponent pulse results in a larger total number of secondary satellites $\left(N_{\text {tot }}=1648\right)$ than for droplet formation driven by the trapezoidal pulse $\left(N_{\text {tot }}=\right.$ 550). Note that the volume distributions are bimodal, with a minimum in the droplet number near a volume of $4 \mathrm{fL}$. Also, note that in particular the number of larger secondary satellites, with a volume greater than or equal to $4 \mathrm{fL}$, is increased when the multicomponent pulse is used instead of the trapezoidal pulse.

To investigate the origin of the bimodality of the secondary-satellite size distributions obtained for the trapezoidal and multicomponent pulses, the axial position of the secondary satellites $y_{s}$ with volumes less than $4 \mathrm{fL}$ is plotted together with that of the secondary satellites with volumes greater than or equal to $4 \mathrm{fL}$. Figure $3(\mathrm{~g})$ shows the result for the trapezoidal pulse, and Fig. 3(h) that for the multicomponent pulse. The nozzle plate is located at $y=0$. Note that for both pulses the satellite droplets located closer to the nozzle are smaller than those closer to the tail droplet.

The difference in the locations of the satellites with a volume less than $4 \mathrm{fL}$ and those with a volume greater than or equal to $4 \mathrm{fL}$ is further investigated by inspecting the secondary tail in more detail around its breakup; see Figs. 3(i) and 3(j). The figures show the stroboscopically imaged formation process of seven different droplets with a temporal resolution of $200 \mathrm{~ns}$, driven by the trapezoidal pulse and by the multicomponent pulse, respectively. Note that the secondary-tail formation is irregular in time. Also, note that for the multicomponent pulse the secondary tail cascades into a tertiary tail, which in some cases leads to the formation of a thick tail droplet on the secondary tail. Rayleigh breakup of the thinner tertiary tail, which occurs notably earlier than breakup of the secondary tail, will produce smaller satellites than those from the secondary tail. Thus, the bimodality and the position dependence of the droplet size distribution for the multicomponent pulse in Figs. 3(f) and 3(h) indeed result from the presence of a thinner tail close to the nozzle, i.e., a tertiary tail. Whether or not a tertiary tail forms for the trapezoidal pulse also is much less evident from the images in Fig. 3(i). However, the bimodality and position dependence of the droplet size distribution for the trapezoidal pulse in Figs. 3(f) and 3(g) show that the secondary tail almost certainly cascades into a tertiary tail for the trapezoidal pulse. Furthermore, it seems like most satellites originate from the tertiary tail.

To shed more light on the formation of a tertiary tail with the trapezoidal pulse, the secondary-tail formation and breakup are imaged with a high-speed camera (Shimadzu HPV-X2) at $5 \mathrm{Mframes} / \mathrm{s}$. This is done for a print head with a $30-\mu \mathrm{m}$-diameter nozzle and ink with a viscosity and surface tension of $10 \mathrm{mPa}$ s and $28 \mathrm{mN} / \mathrm{m}$, respectively. More experimental details are given in the Appendix. The results of this experiment are summarized in Fig. 7, which shows image sequences for eight different droplet formations. Figure 7 clearly demonstrates the formation of a tertiary tail next to the secondary tail for the trapezoidal pulse. In addition, Fig. 7 also reveals the formation of a quaternary tail next to the tertiary tail. The formation of a quaternary tail, and subsequently quaternary satellites, may explain the bimodal distribution in Fig. 3(f) for the satellites with a volume less than $4 \mathrm{fL}$, which has a minimum at approximately $1.5 \mathrm{fL}$. The image sequences in Fig. 7 also show that in most of the eight recordings the satellites originate only from the tertiary and quaternary tails, because the secondary tail contracts and merges with the primary-tail droplet before it can break up. This may explain the relatively low number of satellites with a volume greater than or equal to $4 \mathrm{fL}$ in Fig. 3(f) for the trapezoidal pulse compared with that for the multicomponent pulse.

\section{B. Length of multicomponent pulse}

The delay time $\Delta t$ of the multicomponent pulse is varied from 0.4 up to $14.4 \mu \mathrm{s}$. The different pulse lengths do not alter the head-drop velocity of $6.8 \mathrm{~m} / \mathrm{s}$. The mean number of secondary satellites per droplet formation $\bar{N}_{s}$, the mean secondary-satellite volume $\bar{V}_{s}$, and the mean total secondary-satellite volume per droplet formation $\bar{V}_{t}$ are plotted in Fig. 4(a). The data for measurement series 1, obtained from 1000 droplet formations per data point, are represented by the blue dots, and the data for measurement series 2, obtained from 100 droplet formations per data point, are represented by the red squares. The absolute numbers in the measurement series 1 and 2 are slightly different, but the trend is the same. Data for $\Delta t=2.4 \mu \mathrm{s}$ are not shown, since at this $\Delta t$ a second droplet is ejected after the main droplet.

The mean number of satellites per droplet formation $\bar{N}_{s}$ is at a minimum for the pulse with $\Delta t=9.4 \mu \mathrm{s}$. However, the mean secondary-satellite volume and the mean total secondary-satellite volume per droplet formation are at a minimum at $\Delta t=7.4 \mu \mathrm{s}$. To investigate the observed difference in the locations of the minima in $\bar{N}_{s}, \bar{V}_{s}$, and $\bar{V}_{t}$, the data of the measurement series are plotted again in Fig. 4(b). Now, a distinction is made between the larger 
(a)

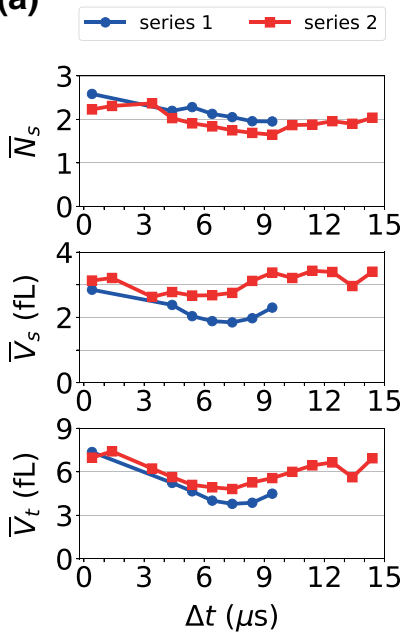

(b) $-v_{s}<4 \mathrm{fL}-v_{s} \geq 4 \mathrm{fL}$ $\rightarrow$ series $1 \quad \longrightarrow$ series 2
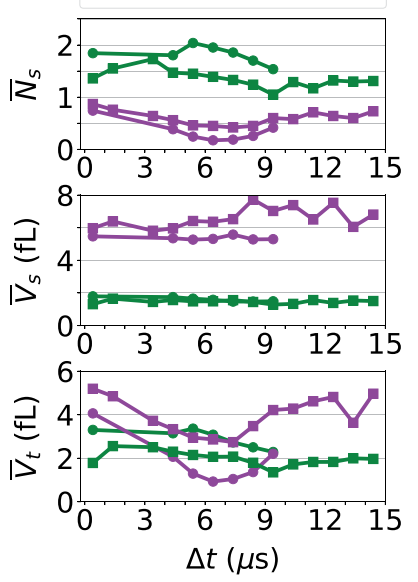

FIG. 4. (a) Mean number of secondary satellites $\bar{N}_{s}$, mean secondary-satellite volume $\bar{V}_{s}$, and mean total secondary-satellite volume $\bar{V}_{t}$ as a function of the delay time $\Delta t$ of the multicomponent pulse. Each data point in series 1 is obtained from 1000 events, while for series 2 this was done for 100 events. (b) Data from (a), where a distinction is made between secondary satellites with $V_{s}<4 \mathrm{fL}$ and with $V_{s} \geq 4 \mathrm{fL}$.

satellites, with a volume greater than or equal to $4 \mathrm{fL}$ (green), and the smaller satellites, with a volume less than $4 \mathrm{fL}$ (magenta). Note that the minimum in $\bar{V}_{t}$ in Fig. 4(a) corresponds to the minimum in $\bar{N}_{s}$ for the larger satellites in Fig. 4(b), which dominate despite their smaller number because of their larger size, while the minimum in $\bar{N}_{s}$ in Fig. 4(a) corresponds to the minimum in $\bar{N}_{s}$ for the smaller satellites in Fig. 4(b), which dominate due to their larger number. This explains the difference between the locations of the minima in $\bar{N}_{s}$ and $\bar{V}_{t}$ in Fig. 4(a).

For multicomponent pulses with $\Delta t=0.4,7.4$, and $9.4 \mu \mathrm{s}$, the secondary-tail formation and breakup are shown in Figs. 5(a) and 5(b). The meniscus position is plotted in Fig. 5(c). The maximum outward meniscus position for the pulses with $\Delta t=7.4$ and $9.4 \mu \mathrm{s}$ is 0.82 times smaller than that for the pulse with $\Delta t=0.4 \mu \mathrm{s}$. Furthermore, in contrast to the situation for the pulse with $\Delta t=$ $0.4 \mu \mathrm{s}$, the meniscus positions of the pulses with $\Delta t=$ $7.4 \mu \mathrm{s}$ and $\Delta t=9.4 \mu \mathrm{s}$ have a second local maximum in the meniscus position. The second local maximum in the meniscus position results in a meniscus velocity that is positive during the start of the formation of the secondary tail, and only slightly negative at the end of its existence; see Fig. 5(d). In contrast, for the pulse with $\Delta t=0.4 \mu \mathrm{s}$, the meniscus velocity is negative, or retracting, over the full lifetime of the secondary tail. The retracting meniscus stabilizes the secondary tail against Rayleigh-Plateau breakup by stretching it $[29,30]$, and therefore most likely causes the $20 \%$ longer secondary tail generated using the pulse

with $\Delta t=0.4 \mu$ s compared with those generated using the pulses with $\Delta t=7.4$ and $9.4 \mu$ s; see Fig. 5(e).

\section{Varying the ink viscosity}

Figures 6(a) and 6(b) show image sequences of secondary-tail formation and breakup for two different ink viscosities, driven by a multicomponent pulse with $\Delta t=$ $0.4 \mu \mathrm{s}$. For an ink viscosity of $5.6 \mathrm{mPa}$ s, the maximum outward meniscus position is 1.25 times larger than that for an ink viscosity of $8.0 \mathrm{mPa}$ s; see Fig. 6(c). The meniscus velocities are very similar for the two ink viscosities; see Fig. 6(d). The secondary-tail length $L_{2 t}$ is plotted in Fig. 6(e) as a function of time. The breakup time of the secondary tail generated using the ink with the lower viscosity occurs $1.7 \mu$ s earlier in time. As a result, the maximum secondary-tail length is $34 \%$ shorter for the low-viscosity ink than for the higher-viscosity ink.

The secondary-satellite volume distributions are plotted in Fig. 6(f). Both distributions are bimodal; note that the change in viscosity does not affect the volume at which the distribution is minimum: this is near a volume of $4 \mathrm{fL}$, as before. Lowering the ink viscosity is observed to lower both the number and the size of the satellites larger than $4 \mathrm{fL}$. Nevertheless, an increase in the number of smaller satellites is observed with a decrease in ink viscosity. All in all, no large difference is observed in the total number of secondary satellites per droplet formation for the two different ink viscosities studied here; these numbers are 1388 and 1530 for ink viscosities of 8.0 and $5.6 \mathrm{mPa} \mathrm{s}$, respectively. However, more importantly, the mean secondarysatellite size and the total secondary-satellite volume both decrease with a decrease in ink viscosity.

\section{DISCUSSION}

First, we discuss the remarkable stability of the submicrometer-diameter secondary tail, which can reach an aspect ratio as large as 40 . Three stabilizing mechanisms are proposed here. Firstly, during secondary-tail formation, a tail droplet with radius $R_{\mathrm{TD}}$ forms on the primary tail. The Laplace pressure in the tail droplet is $2 \gamma / R_{\mathrm{TD}}$, whereas that in the cylindrical secondary tail is $\gamma / R_{2 t}$. Thus, when $2 R_{2 t}>R_{\mathrm{TD}}$, the secondary tail is fed by a flow of liquid from the tail droplet. Secondly, since the secondary-tail diameter is typically smaller than the viscous length scale $\left(L_{\eta}=1.8\right.$ and $0.9 \mu \mathrm{m}$ for the two ink viscosities used in this work), thinning of the secondary tail through the drainage of ink is inhibited. The third stabilizing mechanism results from the retraction of the meniscus towards the nozzle and the contraction of the primary-tail droplet towards the head droplet. Their opposing movement stretches the secondary tail and thereby also the wavelengths of the instabilities in its profile. The faster the meniscus retracts and the primary-tail droplet contracts, the shorter the existence of the instabilities, and, 

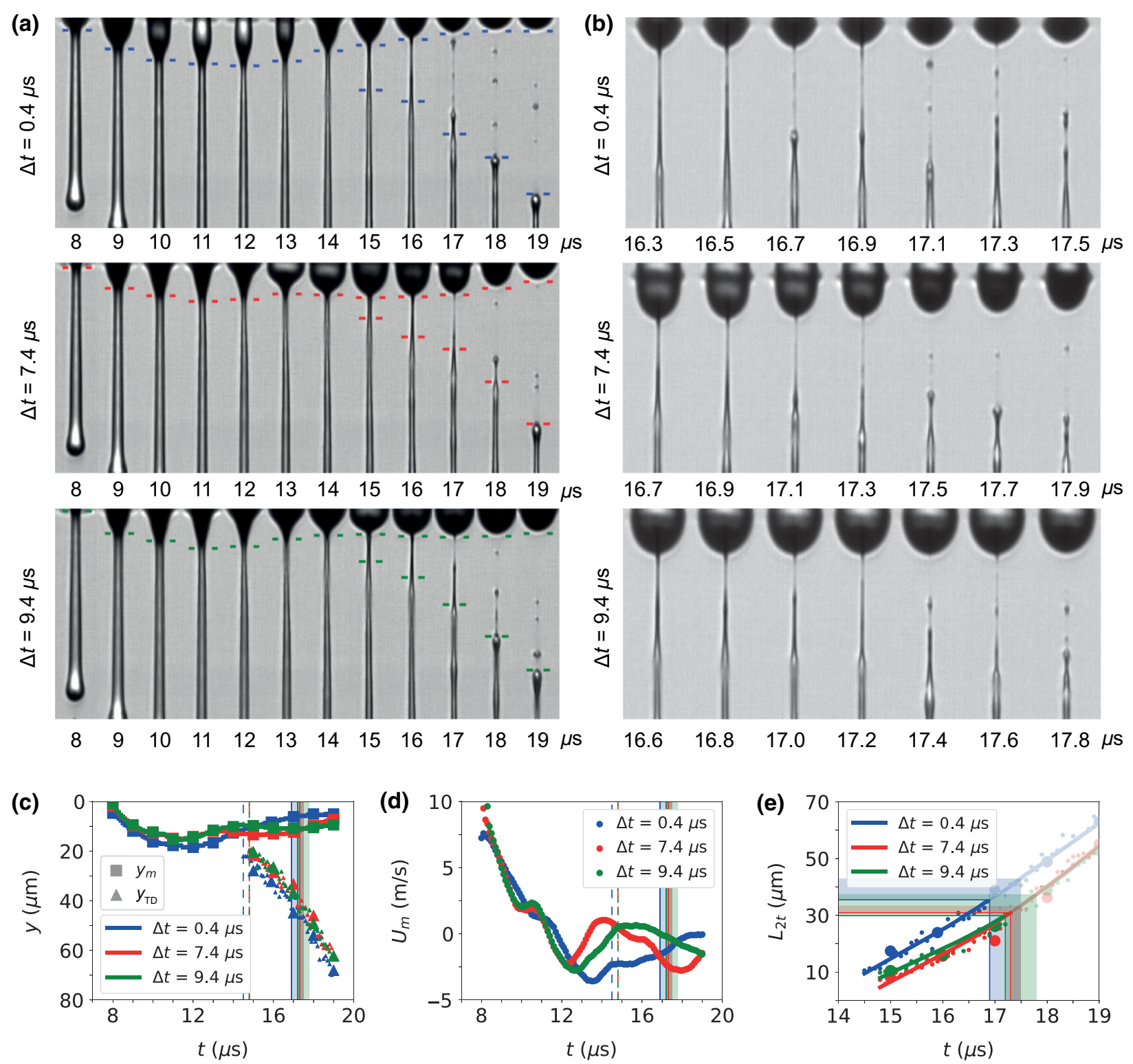

FIG. 5. Secondary-tail formation and breakup for multicomponent pulses with delay times $\Delta t$ of $0.4,7.4$, and $9.4 \mu$ s, (a) with a temporal resolution of $1 \mu \mathrm{s}$, and (b) with a temporal resolution of $0.2 \mu \mathrm{s}$. (c) Meniscus position $y_{m}$ and tail-drop position $y_{\mathrm{TD}}$ as a function of time. The larger symbols in (c),(e) correspond to the images in (a). The dashed and solid vertical lines in (c), (d), and (e) indicate the secondary-tail appearance and breakup, respectively. The translucent bars represent the uncertainty in the breakup time. (d) Meniscus velocity as a function of time. (e) Secondary-tail length as a function of time. After breakup, indicated by the vertical and horizontal solid lines, the evolution of the position of the end of the primary tail is indicated by the translucent data points.

consequently, the more stable the tail is. To determine the relative importance of each mechanism in time, numerical simulations and accurate measurements, in both time and space, of the radial profile of the tail are required.

The observations presented in this paper can be explained using the proposed stabilizing mechanisms. Regarding the driving waveform, the larger secondarytail length observed for drop formation driven by the multicomponent pulse, as compared with that for formation driven by the trapezoidal pulse, can be explained from the 4 times higher negative meniscus velocity (retracting meniscus) observed for the multicomponent pulse, which results in an increased stabilization of the secondary tail through stretching. Regarding the ink viscosity, the measured mean secondary-satellite size decreases with a decrease in the ink viscosity. This decrease may result from a decrease in the viscous length scale. If the secondary-tail thickness scales with the viscous length scale, this will lead to smaller secondary satellites being generated from the secondary tail of an ink jet with a 
(a)

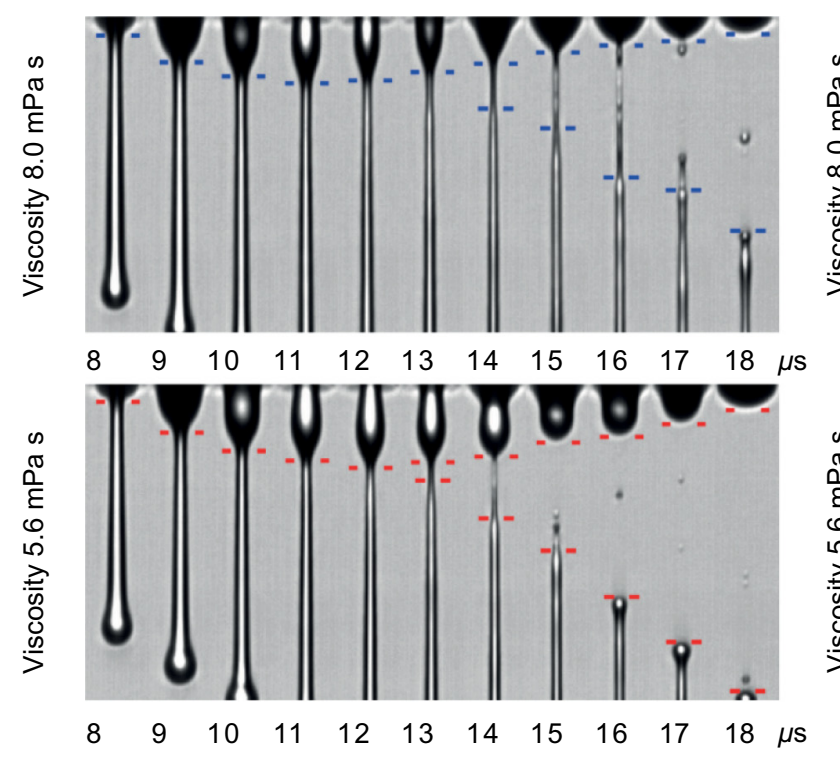

(b)

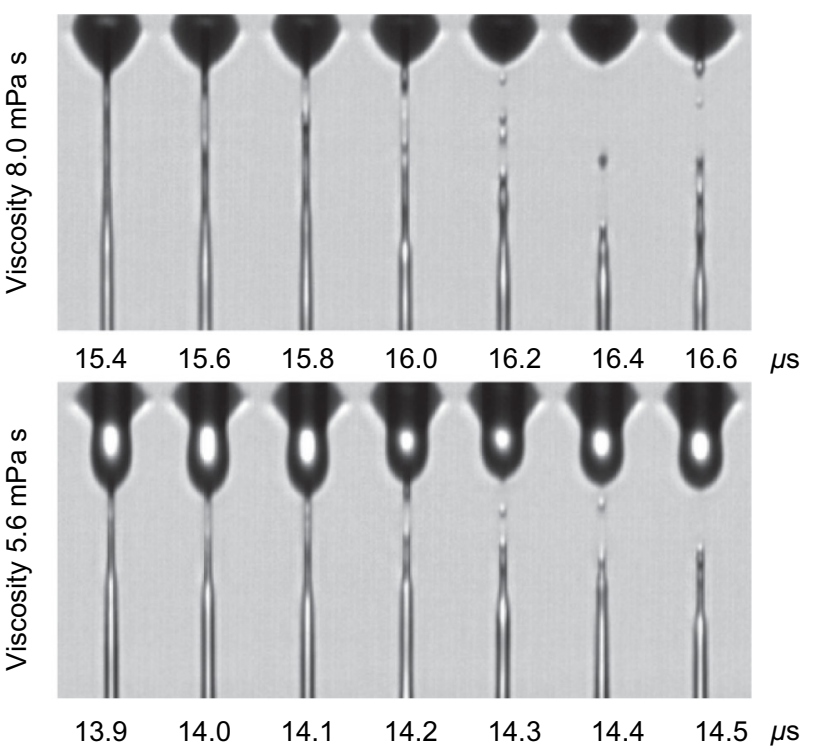

(d)

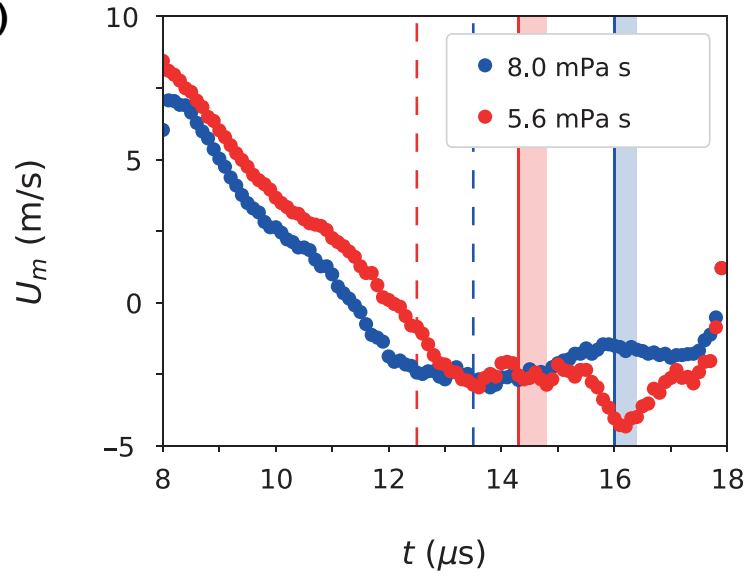

(f)

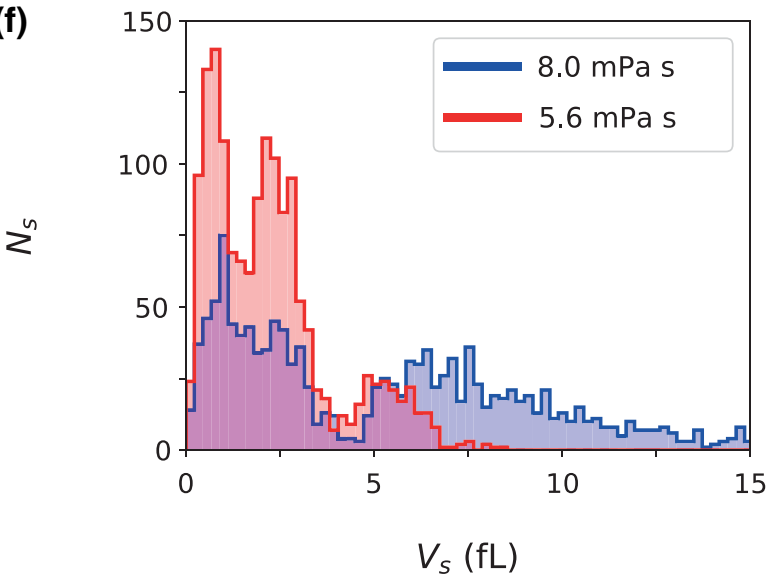

FIG. 6. Secondary-tail formation and breakup driven by a multicomponent pulse with $\Delta t=0.4 \mu$ s for ink viscosities of 8.0 and $5.6 \mathrm{mPa} \mathrm{s}$, (a) with a temporal resolution of $1 \mu \mathrm{s}$, and (b) with temporal resolutions of 0.2 and $0.1 \mu \mathrm{s}$. (c) Meniscus position $y_{m}$ and tail-drop position $y_{\mathrm{TD}}$ as a function of time. The larger symbols in (c),(e) correspond to the images in (a). The dashed and solid vertical lines in (c), (d), and (e) indicate the secondary-tail appearance and breakup, respectively. (d) Meniscus velocity. (e) Secondary-tail length as a function of time. After breakup, indicated by the vertical and horizontal solid lines, the evolution of the position of the end of the primary tail is indicated by the translucent data points. (f) Secondary-satellite volume distributions. 
lower viscosity. The shorter lifetime and lower stability of the secondary tail for the lower ink viscosity result from the reduced viscous damping of the Rayleigh-Plateau instability.

The multicomponent pulses with $\Delta t=7.4 \mu \mathrm{s}$ and $\Delta t=$ $9.4 \mu \mathrm{s}$ result in the same secondary-tail length at breakup. The equal lengths most likely result from the fact that the meniscus is almost stationary in both cases; see Fig. 5(a). The multicomponent pulse with $\Delta t=7.4 \mu \mathrm{s}$ results in a faster meniscus motion towards the nozzle [Fig. 5(d)]; however, this motion sets in only in the final stage of the lifetime of the secondary tail and it therefore does not affect the stability of the secondary tail with respect to that obtained with the multicomponent pulse with $\Delta t=9.4 \mu \mathrm{s}$. Nevertheless, the total number of satellites is larger for the pulse with $\Delta t=7.4 \mu \mathrm{s}$, and the total satellite volume is larger for the pulse with $\Delta t=9.4 \mu \mathrm{s}$. These differences are most likely caused by differences in the radial profile of the tail, most likely due to small differences in meniscus motion and shape, which result in different average length ratios between the secondary and tertiary tails. However, these cannot be resolved, due to the diffractionlimited imaging resolution and the irregularity in time of the secondary- and tertiary-tail formation process.

Future work may focus on numerical modeling of the secondary-tail formation process, potentially allowing a more quantitative understanding of the physical mechanisms that result in secondary- and tertiary-tail formation in drop-on-demand inkjet printing. It would be of interest to address, for example, the cascade in terms of the ratio of the tail thicknesses between successive cascades.

Even though the results in the present work are obtained using a specific in-house-developed print head, the main conclusions, that the stability of the secondary tail decreases with a decrease in stretching rate and in ink viscosity, remain valid for ink jetted from any print head. This acquired understanding can be used to develop new piezodriving waveforms that result in a meniscus that moves towards the secondary tail during its lifetime to minimize its stability, thereby decreasing both its length and its volume at breakup. As a consequence, the number of satellites and the internal contamination of the inkjet printer will be lower. These waveforms can be found for any specific print head through, e.g., an optimization protocol based on a feedback loop that measures the meniscus motion while varying the piezo-driving waveform $[42,43]$. Furthermore, the ink composition can be modified to mitigate satellite formation, e.g., by adding polymers and surfactants [44-47].

\section{CONCLUSIONS}

In this experimental work on droplet formation in dropon-demand inkjet printing, we study the formation of a secondary tail with a diameter on the order of $1 \mu \mathrm{m}$ between the primary tail and the meniscus, in the last microseconds before pinchoff. The influence of the meniscus motion and the liquid viscosity on secondary-tail formation are investigated. We thereby observe the existence of tertiary and quaternary tails in drop-on-demand inkjet printing. The cascade of secondary, tertiary, and quaternary tails breaks up and forms femtoliter satellite droplets. The size distribution of the submicrometer-diameter satellites can be measured in flight using 8-ns laser-induced-fluorescence light pulses. Unlike the formation of the higher-order microthreads, the formation of the secondary tail is highly reproducible, showing that the perturbation leading to secondary-tail formation is not dominated by noise, but by meniscus motion. We demonstrate that the stability of the secondary tail can be controlled through the motion of the meniscus after the primary tail has formed. A 4 times increase in stretching rate results in a 2.2 times increase in the secondary-tail length and a 3 times higher number of femtoliter satellites. Furthermore, a $43 \%$ increase in the ink viscosity is found to increase the secondary-tail length by $50 \%$. The formation of the thicker secondary and thinner higher-order tails results in a bimodal satellite size distribution, where the secondary satellites with a volume greater than or equal to $4 \mathrm{fL}$ are located closer to the primary-tail droplet, while satellites with a volume less than $4 \mathrm{fL}$ are located closer to the nozzle. The highly reproducible cascade leading to a secondary tail is a viable method for verification of the theory of pinchoff dynamics. Furthermore, the inkjet-printing community can benefit from the main conclusions of the present work, that the stability of the secondary tail decreases with a decrease in stretching rate and in ink viscosity, by using them to design waveforms and inks to minimize internal contamination of inkjet printers.

\section{ACKNOWLEDGMENTS}

Maaike Rump is kindly acknowledged for her assistance during the high-speed imaging experiments. This work is part of the "High Tech Systems and Materials" (HTSM) research program, Project No. 12802, and part of the Industrial Partnership Program No. i43, of the Dutch Technology Foundation (STW) and the Foundation for Fundamental Research on Matter (FOM), which are part of the Netherlands Organization for Scientific Research (NWO). The research is cofinanced by Océ Technologies B.V., the University of Twente, and Eindhoven University of Technology.

\section{APPENDIX: HIGH-SPEED RECORDINGS OF TERTIARY-TAIL FORMATION AND BREAKUP}

The formation of a secondary and a tertiary tail in piezoacoustic inkjet printing with a trapezoidal pulse is recorded in more detail using a high-speed imaging setup and a different print head. The ink channel that is used has a Helmholtz resonance frequency of $133 \mathrm{kHz}$, and has a 
cylindrical nozzle with a diameter and length of $30 \mu \mathrm{m}$. As the ink, a noncolored version of the CrystalPoint ${ }^{\mathrm{TM}}$ technology [48] is used, which is a hot-melt ink that is jetted at $130^{\circ} \mathrm{C}$. At this temperature the ink's viscosity, density, and surface tension are $10 \mathrm{mPa} \mathrm{s}, 1080 \mathrm{~kg} / \mathrm{m}^{3}$, and $28 \mathrm{mN} / \mathrm{m}$, respectively. The trapezoidal pulse has rise and fall times of $1.5 \mu \mathrm{s}$, and a high time of $2.0 \mu \mathrm{s}$. The droplets are jetted at a velocity of $9.0 \mathrm{~m} / \mathrm{s}$. The high-speed imaging setup consists of an Olympus transmitted-light microscope with a 50× microscope objective (SLMPLN50x, NA 0.35, working distance $18 \mathrm{~mm}$ ) and a $2 \times$ magnification lens (U-ECA), a Shimadzu HPV-X2 high-speed camera (10 Mframes/s, 256 frames, $400 \times 250$ pixels, $30 \mu \mathrm{m}$ pixel size), and a xenon flash light source, resulting in a spatial resolution of $300 \mathrm{~nm} /$ pixel. The high-speed camera is operated with an exposure time of $110 \mathrm{~ns}$ and a frame rate of $5 \mathrm{Mframes} / \mathrm{s}$. The print head, camera, and light source are triggered with nanosecond precision using a programmable pulse-delay generator (Berkeley Nucleonics Corp., BNC 575). In each experiment, the 1000th droplet formation is imaged.

The formation and breakup of a tertiary tail are recorded for eight different droplet formations. These recordings are summarized in the image sequences shown in Fig. 7. Note that these results not only demonstrate the formation of a tertiary tail during droplet formation with a trapezoidal

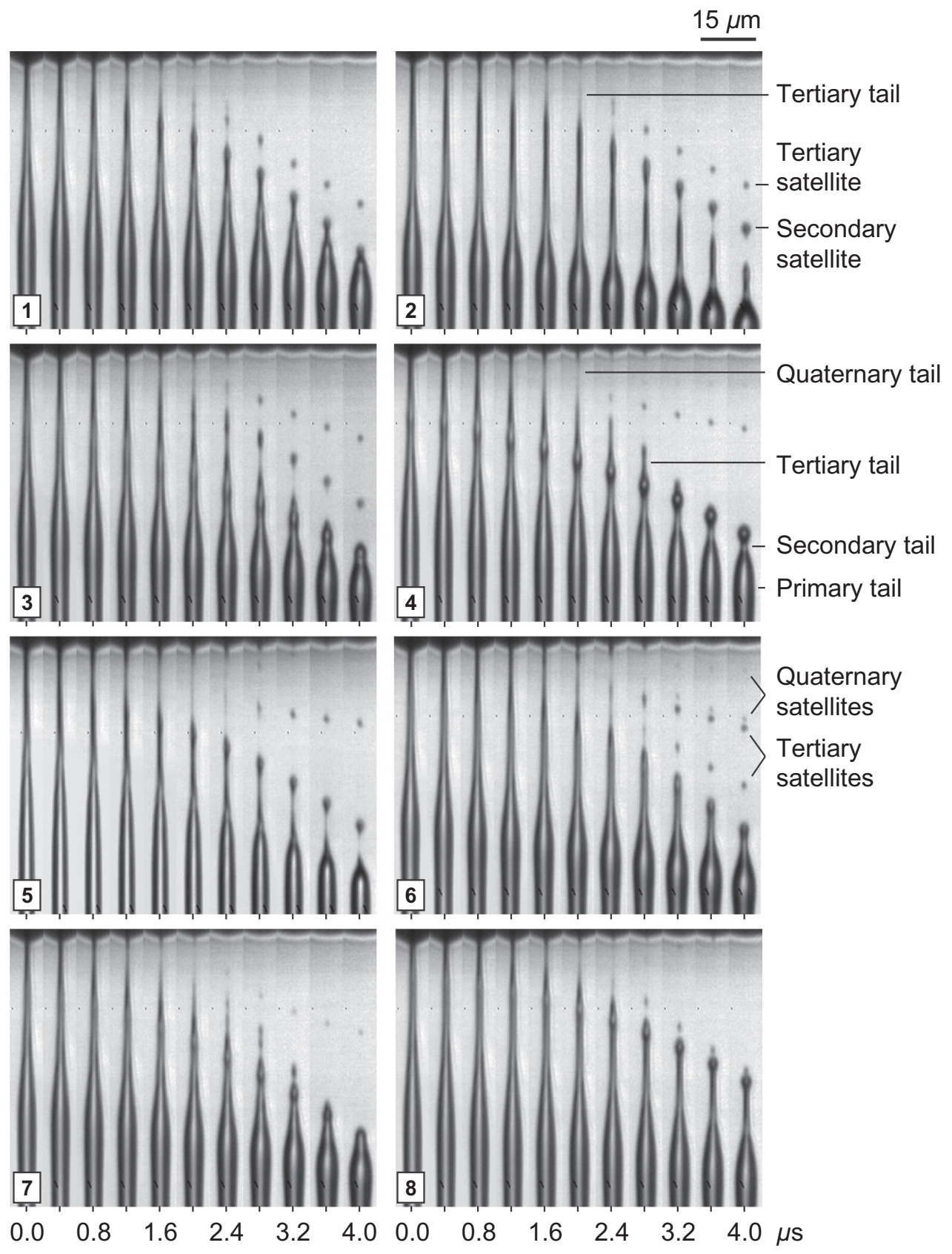

FIG. 7. High-speed recordings of tertiary- and quaternary-tail formation and breakup for eight different droplet formations with a trapezoidal pulse and a $30-\mu \mathrm{m}-$ diameter nozzle. The times given below the image sequences are with respect to the first frame of each image sequence. The temporal resolution is $0.4 \mu \mathrm{s}$. 
pulse, but also reveal the formation of a quaternary tail. Furthermore, note that in most cases the secondary tail contracts into the primary-tail droplet before it can break up, so that only satellites from the tertiary and quaternary tails are left.

[1] H. Wijshoff, The dynamics of the piezo inkjet printhead operation, Phys. Rep. 491, 77 (2010).

[2] S. D. Hoath, Fundamentals of Inkjet Printing: The Science of Inkjet and Droplets (Wiley-VCH Verlag GmbH \& Co. KGaA, Weinheim, Germany, 2015).

[3] O. A. Basaran, Small-scale free surface flows with breakup: Drop formation and emerging applications, AIChE J. 48, 1842 (2002).

[4] J. R. Castrejón-Pita, W. R. S. Baxter, J. Morgan, S. Temple, G. D. Martin, and I. M. Hutchings, Future, opportunities and challenges of inkjet technologies, Atomization Sprays 23, 541 (2013).

[5] T. Shimoda, K. Morii, S. Seki, and H. Kiguchi, Inkjet printing of light-emitting polymer displays, Inkjet Printing Functional Mater. 28, 821 (2003).

[6] C. Jiang, L. Mu, J. Zou, Z. He, Z. Zhong, L. Wang, M. Xu, J. Wang, J. Peng, and Y. Cao, Full-color quantum dots active matrix display fabricated by ink-jet printing, Sci. China Chem. 60, 1349 (2017).

[7] T. M. Eggenhuisen, Y. Galagan, E. W. C. Coenen, W. P. Voorthuijzen, M. W. L Slaats, S. A. Kommeren, S. Shanmuganam, M. J. J. Coenen, R. Andriessen, and W. A. Groen, Digital fabrication of organic solar cells by inkjet printing using non-halogenated solvents, Solar Energy Mater. Solar Cells 134, 364 (2015).

[8] S. G. Hashmi, M. Ozkan, J. Halme, K. D. Misic, S. M. Zakeeruddin, J. Paltakari, M. Grätzel, and P. D. Lund, High performance dye-sensitized solar cells with inkjet printed ionic liquid electrolyte, Nano Energy 17, 206 (2015).

[9] A. Simaite, F. Mesnilgrente, B. Tondu, P. Souères, and C. Bergaud, Towards inkjet printable conducting polymer artifical muscles, Sens. Actuators, B: Chemical 229, 425 (2016).

[10] R. Daly, T. S. Harrington, G. D. Martin, and I. M. Hutchings, Inkjet printing for pharmaceutics - a review of research and manufacturing, Int. J. Pharm. 494, 554 (2015).

[11] S. Hewes, A. D. Wong, and P. C. Searson, Bioprinting microvessels using and inkjet printer, Bioprinting 7, 14 (2017).

[12] M. Nakamura, A. Kobayashi, F. Takagi, A. Watanabe, Y. Hiruma, K. Ohuchi, Y. Iwasaki, M. Horie, I. Morita, and S. Takatani, Biocompatible inkjet printing technique for designed seeding of individual living cells, Tissue Eng. 11, 1658 (2005).

[13] G. Villar, A. D. Graham, and H. Bayley, A tissue-like printed material, Science 340, 48 (2013).

[14] B. Derby, Additive manufacture of ceramic components by inkjet printing, Engineering 1, 113 (2015).

[15] A. U. Chen and O. A. Basaran, A new method for significantly reducing drop radius without reducing nozzle radius in drop-on-demand drop production, Phys. Fluids 14, L1 (2002).
[16] C. Menzel, A. Bibl, and P. Hoisington, MEMS solutions for precision micro-fluidic dispensing application, Technical Report, Fujifilm Dimatix Inc. (2004).

[17] A. van der Bos, T. Segers, R. Jeurissen, M. van den Berg, H. Reinten, H. Wijshoff, M. Versluis, and D. Lohse, Infrared imaging and acoustic sizing of a bubble inside a micro-electro-mechanical system piezo ink channel, J. Appl. Phys. 110, 034503 (2011).

[18] B.-H. Kim, H.-S. Lee, S.-W. Kim, P. Kang, and Y.-S. Park, Hydrodynamic responses of a piezoelectric driven MEMS inkjet print-head, Sens. Actuators, A: Physical 210, 131 (2014).

[19] A. van der Bos, A. Zijlstra, E. Gelderblom, and M. Versluis, iLIF: Illumination by laser-induced fluorescence for single flash imaging on a nanoseconds timescale, Exp. Fluids 51, 1283 (2011).

[20] H. von Helmholtz, On the Sensations of Tone as a Physiological Basis for the Theory of Music (Longmans, Green, and Co., London, New York, 1895).

[21] J. R. Castrejón-Pita, G. D. Martin, S. D. Hoath, and I. M. Hutchings, A simple large-scale droplet generator for studies of inkjet printing, Rev. Sci. Instrum. 79, 075108 (2008).

[22] J. R. Castrejón-Pita, A. A. Castrejón-Pita, S. S. Thete, K. Sambath, I. M. Hutchings, J. Hinch, J. R. Lister, and O. A. Basaran, Plethora of transitions during breakup of liquid filaments, Proc. Natl. Acad. Sci. 112, 4582 (2015).

[23] N. Morita, T. Hamazaki, and T. Ishiyama, Observation on satellite behavior by double-pulse driving for high-speed inkjet, J. Imaging Sci. Technol. 60, 40503 (2016).

[24] H. Y. Gan, X. Shan, T. Eriksson, B. K. Lok, and Y. C. Lam, Reduction of droplet volume by controlling actuating waveforms in inkjet printing for micro-pattern formation, J. Micromech. Microeng. 19, 055010 (2009).

[25] H. Dong, W. W. Carr, and J. F. Morris, An experimental study of drop-on-demand drop formation, Phys. Fluids 18, 072102 (2006).

[26] H. M. A. Wijshoff, Drop formation mechanisms in piezo-acoustic inkjet, Proc. Nanotech 2007 3, 448 (2007).

[27] Q. Xu and O. A. Basaran, Computational analysis of drop-on-demand drop formation, Phys. Fluids 19, 102111 (2007).

[28] A. van der Bos, M.-J. van der Meulen, T. Driessen, M. van den Berg, H. Reinten, H. Wijshoff, M. Versluis, and D. Lohse, Velocity Profile Inside Piezoacoustic Inkjet Droplets in Flight: Comparison Between Experiment and Numerical Simulation, Phys. Rev. Appl. 1, 014004 (2014).

[29] I. Frankel and D. Weihs, Stability of a capillary jet with linearly increasing axial velocity (with application to shaped charges), J. Fluid Mech. 155, 289 (1985).

[30] S. Le Dizès and E. Villermaux, Capillary jet breakup by noise amplification, J. Fluid Mech. 810, 281 (2017).

[31] T. A. Kowaleski, On the separation of droplets from a liquid jet, Fluid Dyn. Res. 17, 121 (1996).

[32] X. D. Shi, M. P. Brenner, and S. R. Nagel, A cascade of structure in a drop falling from a faucet, Science 265, 219 (1994). 
[33] M. P. Brenner, X. D. Shi, and S. R. Nagel, Iterated Instabilities During Droplet Fission, Phys. Rev. Lett. 73, 3391 (1994).

[34] J. Eggers, Universal Pinching of 3D Axisymmetric FreeSurface Flow, Phys. Rev. Lett. 71, 3458 (1993).

[35] M. Rubio, A. Ponce-Torres, E. J. Vega, M. A. Herrada, and J. M. Montanero, Complex behavior very close to the pinching of a liquid free surface, Phys. Rev. Fluids 4 , 021602 (2019).

[36] P. T. McGough and O. A. Basaran, Repeated Formation of Fluid Threads in Breakup of a Surfactant-Covered Jet, Phys. Rev. Lett. 96, 054502 (2006).

[37] P. M. Kamat, B. W. Wagoner, S. S. Thete, and O. A. Basaran, Role of marangoni stress during breakup of surfactant-covered liquid threads: Reduced rates of thinning and microthread cascades, Phys. Rev. Fluids 3, 043602 (2018).

[38] M. P. Brenner, J. R. Lister, and H. A. Stone, Pinching threads, singularities and the number $0.0304 \ldots$, Phys. Fluids 8, 2827 (1996).

[39] J. Eggers and E. Villermaux, Physics of liquid jets, Rep. Prog. Phys. 71, 036601 (2008).

[40] Python Software Foundation, https://www.python.org/.
[41] H. C. van de Hulst, Light Scattering by Small Particles (Dover Publications Inc., New York, 1981).

[42] A. A. Khalate, X. Bombois, G. Scorletti, R. Babuska, S. Koekebakker, and W. de Zeeuw, A waveform design method for a piezo inkjet printhead based on robust feedforward control, J. Microelectromech. Syst. 21, 1365 (2012)

[43] K.-S. Kwon, Waveform design methods for piezo inkjet dispensers based on measured meniscus motion, J. Microelectromech. Syst. 18, 1118 (2009).

[44] O. A. Basaran, H. Gao, and P. P. Bhat, Nonstandard inkjets, Annu. Rev. Fluid Mech. 45, 85 (2013).

[45] N. F. Morrison and O. G. Harlen, Viscoelasticity in inkjet printing, Rheol. Acta 49, 619 (2010).

[46] G. D. Martin, S. D. Hoath, and I. M. Hutchings, Inkjet printing - the physics of manipulating liquid jets and drops, J. Phys.: Conf. Ser. 105, 012001 (2008).

[47] S. D. Hoath, S. Jung, W.-K. Hsiao, and I. M. Hutchings, How PEDOT:PSS solutions produce satellite-free inkjets, Org. Electron. 13, 3259 (2012).

[48] Océ Technologies B.V. For more information on Océ CrystalPoint technology see https://www.oce.com/products/ crystalpoint/. 